\title{
A Robotic Sensor Network for Monitoring Carp in Minnesota Lakes
}

\author{
Deepak Bhadauria, Volkan Isler, Andrew Studenski and Pratap Tokekar
}

\begin{abstract}
Robotic Sensor Networks (RSNs) find increasing use in environmental monitoring as RSNs can collect data from obscure, hard-to-reach places over long periods of time. This work reports progress in building a network of small, lightweight robotic rafts which will be used to monitor common carp tagged with radio transmitters across Minnesota lakes. We describe the design and architecture of the robotic raft, and demonstrate the robustness of our waypoint navigation algorithm through field tests conducted in various lakes. We also present results from experiments aimed towards localizing tagged fish.
\end{abstract}

\section{INTRODUCTION}

A Wireless Sensor Network (WSN) is a network of inexpensive, low-power computing devices with sensing and wireless communication capabilities. WSN research has been very active in the last two decades with researchers focusing on issues such as the development of energy-efficient communication protocols to improve network lifetime [1]. Consequently, the technology advanced to the level that WSNs are now being routinely used in environmental applications such as the monitoring of humidity levels to determine vineyard irrigation levels [2].

Environmental monitoring is an important application domain for WSN technology as a WSN enables collection of data at unprecedented spatial and temporal scales. Unfortunately, it is very difficult to deploy and maintain networks of stationary sensors over large areas. As an example, consider the task of monitoring carp in Minnesota's 10,000+ lakes. These lakes vary in size and some are interconnected, forming complex interactions.

The common carp is recognized as an invasive species to the waters of the Midwestern United States. Carp pose a significant threat to natural ecosystems due to the large quantities of harmful nutrients which they release while bottom-feeding. It is for this reason that understanding and monitoring carp populations has garnered increased interest in areas with significant carp infestations, such as the lakes of Minnesota. Professor Peter Sorensen, a leading expert of fish behavior with the Department of Fisheries, Wildlife, and Conservation Biology at the University of Minnesota, is dedicated to tracking and controlling the species.

In order to study fish behavior, Dr. Sorensen's team tag carp with radio transmitters. The fish are caught and transmitters are surgically inserted under their skin before they are introduced back into the lake. These tags emit short

The authors are listed in alphabetical order. The corresponding author is Pratap Tokekar. All authors are with the Department of Computer Science, University of Minnesota, Minneapolis, MN, 55455, USA Emails: \{bhadau, isler, studen, tokekar\}ecs.umn.edu

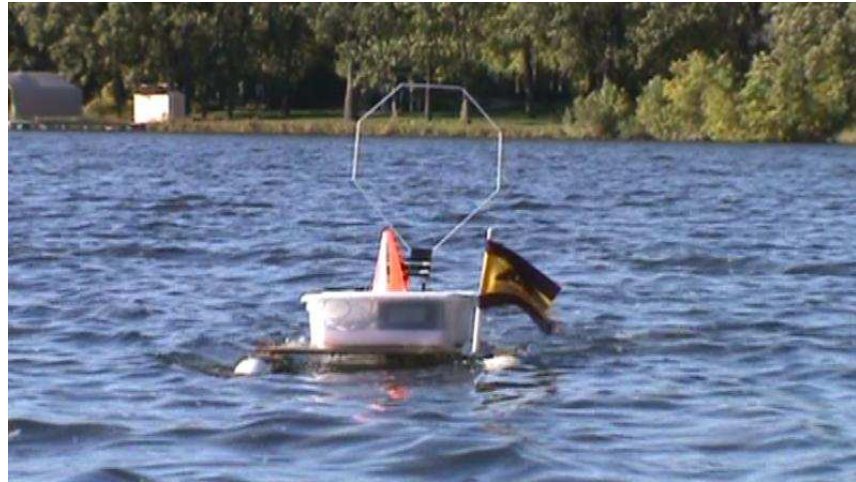

Fig. 1. The raft during field trials at Lake Phalen.

regular pulses which can be detected using a directional antenna up to 50 meters under ideal conditions. Collecting data then requires the work of two lab members: one to steer a boat toward locations where the fish are likely to be found, and the other to operate the antenna. The process of actually locating a fish requires the latter lab member to rotate a directional antenna, give directions to the other lab member and record measurements from GPS and antenna simultaneously. Consequently, data collection is usually imperfect and can be performed only for a limited duration. Yet, Dr. Sorensen's group is often interested in determining carp distributions at obscure places and times such as shallow wetlands where carp can migrate and reproduce. Sometimes the data is required to be collected at odd hours, for example during daybreak, a time which is prohibitively cold in Minnesota weather. The ability to continuously monitor the lakes without manual involvement would thus prove useful.

We are collaborating with Dr. Sorensen's group to automate the data collection process. At first, one might think that a network of stationary antenna would be suitable for this task. However, a data logger, receiver and antenna combination costs about $\$ 3,000$, and has a range of roughly 50 meters. Therefore, even covering a single lake could be costly. Further, deploying such networks across numerous interconnected lakes around the Twin Cities would be prohibitively costly. We believe that a network of a small number of light-weight robotic rafts could be ideal for this task. Such a network can be easily deployed and it can autonomously reconfigure itself based on the location of the tagged fish. We recently started building a robotic raft for monitoring carp (Figure 1). In this paper, we present our current design, and report results from the first set of field experiments which demonstrate the utility of the system. 
The rest of the paper is organized as follows: The related work is presented in Section II. Section III gives details about the raft's hardware design and system architecture. The navigation algorithm is described in Section IV and the field experiments are presented in Section V. Finally, we conclude by presenting an overview of future work in Section VI.

\section{RELATED WORK}

Marine robotics has seen significant activity recently. Numerous groups across the world are involved in designing and developing robots which can navigate in water bodies and perform various data collection tasks. An Autonomous Surface Vehicle (ASV) named ROAZ [3] was developed for operation in rivers and estuaries. The main objective was to perform aquatic environmental monitoring and to support operations of autonomous underwater vehicles (AUV). Another ASV was developed at Virginia Tech [4] for mapping shorelines. Researchers at University of South Florida developed unmanned surface vehicle with autonomous and teleoperated control [5] for testing and deploying environmental and oceanographic instrumentation. Additionally, underwater autonomous systems have garnered a significant amount of attention and the designs of such systems are improving rapidly [6]. There are many systems already developed or in use for tasks like environmental monitoring [7], [8], oceanography [9], [10], and autonomous intervention [11].

Our system has two primary design constraints. Minnesota has a large number of lakes interconnected through streams and rivers. Researchers desire to track carp across the entire watershed, therefore the system should be rapidly deployable, and thus be small and lightweight (ideally it should fit within a car). Second, the cost of the system should be low, so that we can build multiple such rafts. Most of the systems mentioned above are large in size (in fact, they require special deployment equipment) and expensive.

The system described in [12] is closest to our system in design. It consists of a number of stationary buoys deployed in a lake along with a robotic boat capable of autonomous navigation. The buoys continuously monitor the environment and communicate collected sensor information to the boat. The boat also samples the water body using onboard sensors. Our system differs from this system in that, as previously mentioned, we cannot deploy static nodes as the cost scales to prohibitive levels in larger implementations. Additionally, instead of collecting environmental data relative to the lake itself, we focus on mobile entities inside the lake for which new searching and tracking algorithms must be developed.

\section{DESIGN}

The following subsections describe the design goals and the resulting implementation for the main components of the system.

\section{A. Radio Tags, Receiver and Data Logger}

Dr. Sorensen's group use the radio tag equipment manufactured by Advanced Telemetry Systems (ATS). The complete system consists of radio tags, a loop antenna connected

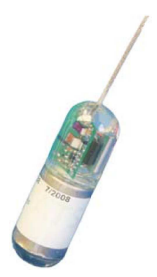

Fig. 2. The transmitter used for tagging fish is $85 \mathrm{~mm}$ long and $15 \mathrm{~mm}$ in diameter. Image from ATS Track.

to a Radio Receiver and a Data Logger which provides the computer interface for the Receiver. The radio tags shown in Figure 2 are $85 \mathrm{~mm}$ long and $15 \mathrm{~mm}$ in diameter and have a trailing whip antenna. They use internal lithium battery as the power source. To conserve power, these tags typically emit a pulse for $20 \mathrm{~ms}$ every $1100 \mathrm{~ms}$. Each tag emits a single frequency (in the range 48-50 MHz), hence, when multiple tags are to be used within the same lake, tags with different unique frequencies are used. Since each tag emits a pulse every $1100 \mathrm{~ms}$, the antenna has to continuously scan for this frequency for a time greater than $1100 \mathrm{~ms}$ to detect the presence/absence of the tag, before moving on to the next frequency.

A directional loop antenna is used to detect the signal emitted by the radio tags. The received signal strength is highest when the tag is aligned with the plane of the antenna. It is lowest when the antenna is perpendicular to the direction of tag and decreases along the way. Hence, we can estimate the bearing of the tag by panning the antenna in a complete circle and noting the signal strength readings. The direction with the maximum signal strength reading points towards the location of the tag. The antenna shows identical signal strength characteristics if the tag lies on either side of the antenna along a straight line, and hence only a scan of $180^{\circ}$ is required.

The Data Logger (D5401A) provides a programmable interface between the Receiver (R2100) and an on-board laptop. The Data Logger has memory for four frequency tables each capable of storing up to 100 frequencies. A preset frequency list to be scanned can be entered into the table, depending upon the lake. The scanning interval of the Receiver can also be set using the Data Logger. Once the Receiver is enabled, the Data Logger stores the data in its memory. The stored data includes scanning frequency, received signal strength and a time stamp.

\section{B. Physical System Design}

Development of the physical system was largely constrained by the system used to track carp which was already operating in the field. To make use of the directionality of the antenna it is mounted on a pan-tilt unit. To ensure stability of the pan-tilt unit and the payload (Receiver and Data Logger) we decided to use a single catamaran style craft as shown in Figure 3. For transportation purposes the raft was designed to be light-weight and sturdy as well as small enough to fit inside a regular-sized automobile. In order to keep the cost of the raft at a minimum, existing 


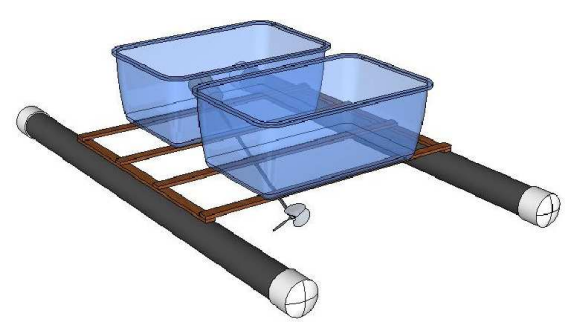

Fig. 3. 3D model of the raft: The raft has a length of 5 feet and width of 3 feet.

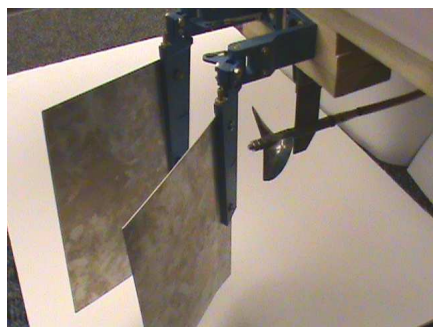

Fig. 4. Rudder and Propeller assembly: The two rudders are connected together to a servo motor. The propeller connects to DC motor through a flexible shaft.

commercially available parts were used. Two 5 feet sections of 4 inch PVC pipes are securely fastened on a thin wooden frame. The electronics and on-board laptops are placed inside two plastic bins placed over the frame. We use a 3 inch diameter 3-blade propeller attached to a 12 volt DC motor through a flexible shaft for the propulsion of the raft, shown in Figure 4. The steering comes from two modified hobby boat rudder assembly connected to a single servo motor. A 12 volt, $18 \mathrm{Ah}$ sealed lead-acid battery provides 5 hours of continuous operation.

\section{System Architecture}

The system architecture was developed keeping in mind the following design goals and requirements:

Modular design: High-level functionality such as navigation and data logging are separated from low-level device control such as driving the propeller and steering. This allows flexibility in adding new components to the system and modifying the existing ones without affecting other components.

Remote access: User should be able to monitor the data collection and set navigation waypoints for the raft remotely. In metro area lakes, often wireless Internet access is available. The raft can connect to the Internet which allows remote operation and visualization.

Optional manual override: selective radio control over steering and propulsion in case of emergency situations.

With these goals in mind, a two-level system was devised (see Figure 5): low-level control is provided by the Robostix 8-bit microcontroller board while high-level software runs on an on-board netbook. The Robostix connects to the drivers for the propeller and rudder motors. The antenna is mounted on a pan-tilt unit to make use of the directionality of the antenna. The Robostix runs the low-level control software for

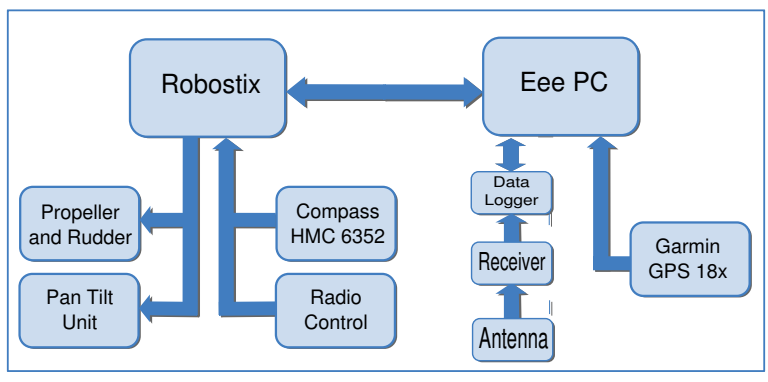

Fig. 5. Overall System Architecture: The low-level control software runs on the Robostix microcontroller. The Eee PC controls the radio antenna and runs the high-level navigation routines.

the pan-tilt unit. A 4-channel FM radio controller (used in RC helicopters) is used to provide optional manual override in case of emergency. The on-board netbook interfaces with the Data Logger and antenna equipment. We use GPS sensor and a digital compass for autonomous navigation. We describe each of these components in the following subsections.

1) Eee PC: The Eee PC netbook from ASUS, running Linux, is the main computer on the raft. The software running on the Eee PC manages high level motion planning and continuously reads all the sensors. GPS information is collected directly from the Garmin 18x GPS unit connected via USB. Data from the radio antenna and receiver is recorded via the Data Logger unit over USB. The compass readings are obtained from the Robostix unit at regular intervals during execution. We can connect to the on-board Eee PC over a remote ssh connection using another laptop from the shore. This allows us to monitor the current data or modify the running program remotely without removing the raft from the water.

2) GPS: The Garmin 18x GPS receiver interfaces with the Eee PC through a USB port. The GPS refreshes and receives a new reading once every second. The WAAS-enabled GPS is rated for error less than 3 meters. In addition to location information, the GPS also transmits the velocity in the North and East directions. The heading of the robot, $H_{g p s}$, can be calculated using the velocity values using,

$$
H_{\text {gps }}=\tan ^{-1}\left(\frac{v_{N}}{v_{E}}\right)
$$

where $v_{N}$ and $v_{E}$ are the velocity values in North and East directions obtained from GPS.

3) Microcontroller: The Robostix is a commerciallyavailable, ATMEL ATMega128 based microcontroller board from Gumstix. It generates signals to drive the propeller motor and rudder servo motor taking input commands from the Eee PC. It also reads the current heading of the raft from the digital compass using the $\mathrm{I} 2 \mathrm{C}$ protocol. Additionally, the Robostix controls the pan and tilt servo motors on which the antenna is mounted. A separate $7.2 \mathrm{~V} 4200 \mathrm{mAh} \mathrm{NiMh}$ battery is used to power the Robostix and the compass.

As a safety feature, three channels from the 4-channel FM receiver are connected to Robostix. If the "Override" channel is active, commands from the Eee PC are ignored and the user can directly control the propeller and rudder using the 
other two channels.

4) Compass: The Honeywell HMC6352 compass module from SparkFun Electronics is used on the raft to provide the heading angle information. The compass is rated to give heading resolution of $0.5^{\circ} / \mathrm{s}$ and an accuracy of $2.5^{\circ} / \mathrm{s}$. The compass combines two magneto-resistive sensors to sense the horizontal components of earth's magnetic field to compute the heading information.

\section{NAVigation Algorithms}

The raft uses on-board GPS and compass sensors as feedback for navigation. GPS gives the position in terms of latitude and longitude and the heading of the raft can be obtained from the compass and the GPS velocity values. Our initial testing of the GPS revealed that the error in velocity values is very large when the raft is stationary. However, when the GPS is moving, the magnitude of error reduces and the velocity values are much more reliable. The digital compass, on the other hand, is affected by magnetic fields in its vicinity, including those generated by the on-board electronic circuitry. Hence instead of relying on only one sensor, for computing the heading of the raft $H_{\text {heading }}$, we take a weighted average of the compass reading $H_{\text {comp }}$ and the heading obtained from GPS velocity $H_{g p s}$.

$$
H_{\text {heading }}=\beta H_{\text {comp }}+(1-\beta) H_{\text {gps }} .
$$

where $\beta$ is the weighting factor, which can be set as per the confidence for each sensor. A probabilistic filter can be used instead of weighted average, for combining the two sensor readings. The weighted average approach, however, does not require the knowledge of error distribution of GPS and compass. We found that the weighted average approach works effectively for a wide choice of weights. The position information is directly obtained from the GPS.

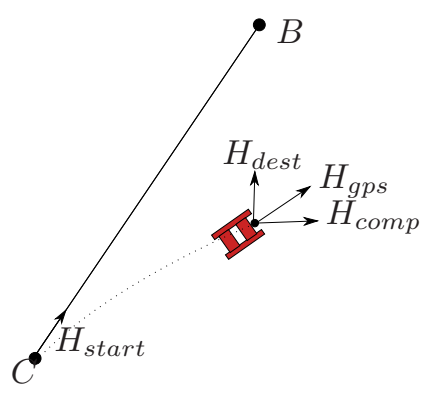

Fig. 6. Waypoint navigation between points $A$ and $B$ : $H_{g p s}$ and $H_{c o m p}$ are the headings obtained from the GPS and compass respectively. $H_{c o m p}$ is shown with slight error with respect to true heading of the raft. $H_{\text {dest }}$ is the desired heading.

A simple proportional controller is used to generate the steering angle $\theta$ for the raft. While going from starting point $A$ to destination point $B$, the angle by which the raft should steer depends upon the current heading of the raft $H_{\text {heading }}$ (given in Equation 1), the angle made by the line $A B$, denoted by $H_{\text {start }}$ and the angle made by the line joining the current position of the raft to the destination position, called $H_{\text {dest }}$. The desired change in the heading, $\Delta H$, of the raft is then given by,

$$
\begin{gathered}
\Delta H=\alpha\left(H_{\text {start }}-H_{\text {heading }}\right)+(1-\alpha)\left(H_{\text {dest }}-H_{\text {start }}\right) \\
\theta=k_{P} \Delta H
\end{gathered}
$$

where $\alpha$ is a weighting factor. The first term in Equation 2 gives the error between the starting heading and the current heading, whereas the second term calculates the error between the current heading and the desired heading. The steering angle $\theta$ is set proportional to the error $\Delta H$. The weighting factors $\alpha, \beta$ and the constant of proportionality, $k_{P}$ can be determined experimentally. During the experiments, it was found that the GPS and compass readings occasionally had large errors. However, these errors did not last long. For example, when the raft is stationary, the GPS heading values have high error. The error reduces significantly as soon as the raft starts moving. As a result, changing the weighting factors does not change the behavior of the system significantly in the long run. We set the values of $\alpha$ and $\beta$ to be 0.5 each, after initial testing.

The waypoint navigation algorithm was designed so as to perform in the case of the raft drifting from its course due to waves from other boats in the lake and wind. Such waves and wind can potentially throw the raft off the straight line which would require large error correction. To deal with such cases, we constantly check to see if the raft is within a particular band drawn about the line $A B$. If the raft drifts outside of this band on either side, we make a new call to the method with the current position and heading of the raft as the starting point towards the same destination.

We call the above method repeatedly when navigating on a series of waypoints, for each consecutive pair of waypoints. Since the GPS location information has some error, we check if the raft has reached the destination by checking if it is within a certain radius (set to 3 meters, the rated error of GPS) from it. The algorithm terminates when the raft reaches the final waypoint.

\section{FIELD EXPERIMENTS}

We conducted several trials in Spoon Lake, Lake Keller, Lake Riley and Lake Phalen around Minneapolis, MN to test autonomous waypoint navigation and control of the raft. The details about these trials is presented below.

\section{A. Autonomous Waypoint Navigation}

The trials for testing autonomous waypoint navigation were performed in Lake Keller in Maplewood, MN. This lake is about 966 meters along its length. The waypoint navigation algorithm was given a series of GPS coordinates within the lake to visit in sequence for each trial. The results for two such trials conducted in different parts of the lake are shown in Figure 7. For the left GPS trail shown in Figure 7, labels $P 1$ to $P 5$ were the waypoints to be visited by the raft. The total distance covered by the raft for this trial was about $304 \mathrm{~m}$. For the right GPS trail in Figure 7, the raft was given the waypoints $P 1$ to $P 6$ to follow, before coming back to $P 1$ again. The motion between two successive waypoints 

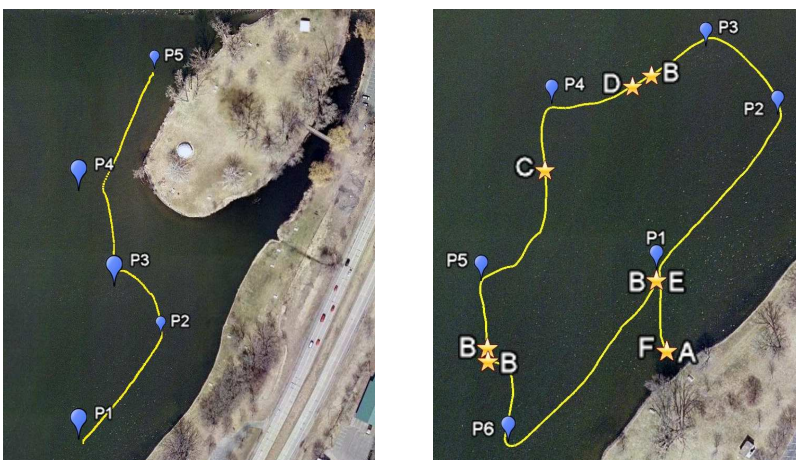

Fig. 7. Field Experiments at Lake Keller. Labels $P_{1}$ to $P_{6}$ are waypoints. Star marks location of raft where tagged fish were detected. The total path length was about $304 \mathrm{~m}$ and $540 \mathrm{~m}$ for the left and right figures respectively.

is smooth and straight most of the times. While moving between waypoints $P 4$ to $P 5$ and $P 5$ to $P 6$ the raft moved away from the straight line initially. This could be attributed to large waves from other boats in the lake causing the raft to drift aside. However, the correction routine checked that the raft was outside of the defined band and ensured that the raft stayed on course. The total distance traveled by the raft in this trial was about 540 meters which was covered in 16 minutes.

We conducted more such trials for testing the waypoint navigation algorithm. The raft successfully completed the trials in each case in a robust fashion. All the experiments were conducted in natural, uncontrolled environment with other boats operating in the vicinity of the raft.

\section{B. Fish Detection}

During the trials for testing the waypoint navigation algorithm, we also tested the radio receiver. There are 22 fish in Lake Keller tagged with radio transmitters. The data logger was configured to scan for all the 22 frequencies and record the data while performing the trials. A number of tags were detected during these trials. The location of the raft where the tags were detected is marked with star in the right trail in Figure 7. The labels on the stars correspond to the frequency of the radio tag; each label corresponds to a distinct frequency detected at multiple locations.

During one of the early trials, the raft was left stationary at a location for about 30 minutes. During this time, a total of 5 different frequency tags were detected, out of which the tag with frequency $48.691 \mathrm{MHz}$ was detected thrice for 5 minutes and was not detected at later times. This suggests that the fish were moving in this period and would require sophisticated strategy to track and localize them.

These results demonstrate the capability of the system to detect the presence and absence of tagged fish in the radio range of the antenna. While this is certainly useful in finding which part of the lake the fish are present, the radio range could be as large as 50 meters. Our main objective is to be able to precisely locate these fish. To achieve this, it is important to understand the behavior and characteristics of the radio tags and antenna.

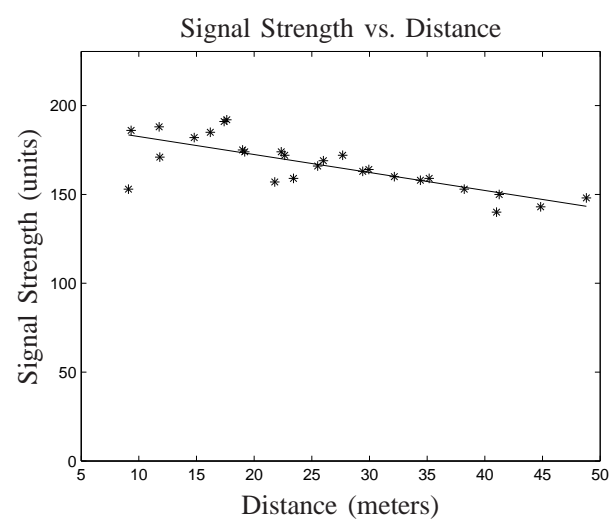

Fig. 8. Plot of signal strength vs. distance with least squares linear fit. Depth: 1m, Range: $49 \mathrm{~m}$.

\section{Fish Localization}

In addition to detecting the signals from the radio transmitters, the radio receiver provides the signal strength measured by the antenna for that signal. As previously mentioned, the antenna used on the raft to detect the signal is directional. The signal strength is maximum when the antenna is directly pointing towards the tag. This signal strength and bearing information can be used to calculate a more precise location of a tagged fish.

1) Using Signal Strength: To understand the relationship between signal strength and detection distance, we conducted a set of experiments at Lake Riley in Eden Prairie, Minnesota. A reference frequency tag was kept under water in the middle of the lake at a depth of about 1 meter. The raft was steered in a straight line away from the tag, with the antenna was always pointing towards the tag (i.e. in the direction of maximum signal strength). The plot of the observed readings with respect to distance is shown in Figure 8. It can be observed that the the signal strength decreases with respect to distance, in general. The maximum distance from the tag up to which the signal was received for this trial was approximately 49 meters. However, this relation is also a function of the depth of the tag in the water. We repeated the same experiment varying the tag depth to 2 and 4 meters. For the depth of 2 meters, the tag was only detected up to a distance of 20 meters, while for the depth of 4 meters, this distance further reduced to 10 meters. This makes performing localization using just signal strength measurement from one location very difficult as the depth of the fish is not known. It is clear from these results, that signal strength alone cannot be used to localize the fish.

2) Triangulation using Bearing: Since the directional antenna provides bearing information, we can use bearings from multiple locations to localize the fish. We performed a triangulation experiment with the reference tag in Lake Keller. The reference tag was suspended in the water at the location marked "Tag" in Figure 9. The raft visited three pre-defined locations marked $A, B$ and $C$ in sequence. At each location, the pan unit was instructed to rotate from $0^{\circ}$ to $180^{\circ}$ in steps of $45^{\circ}$. Since the antenna response is 
symmetric along its plane, these readings make a complete sweep in all directions. Out of these readings, the bearing with maximum signal strength is chosen as the direction towards the tag. Using the 3 bearings obtained from $A, B$ and $C$ we can perform triangulation to calculate the location of the tag. The triangulation performed for one such trial is

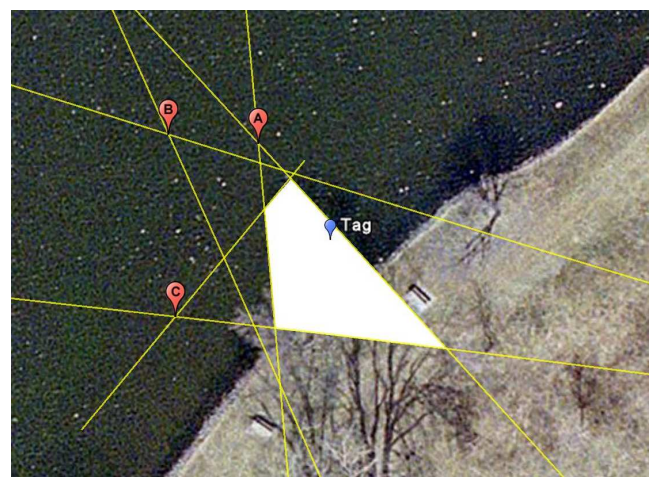

Fig. 9. Triangulation experiment: Cones of $45^{\circ}$ drawn around direction of maximum signal strength at locations $A, B$ and $C$. Their intersecting polygon whose interior consists of possible location of the tag. The tag was actually placed at the location marked "Tag".

shown in Figure 9. The true location of the tag is marked in the figure by "Tag". The readings were obtained in steps of $45^{\circ}$ and hence at each location we obtain a cone $\left(-22.5^{\circ}\right.$ to $\left.+22.5^{\circ}\right)$ rather than a single line. Their intersection results in a polygon, the interior of which denotes the possible location of the tag. The polygon shown in Figure 9 has a total area of about $176 \mathrm{~m}^{2}$. At present, the locations for points $A, B$ and $C$ are pre-defined. The area of intersection can be reduced by choosing better locations for $A, B$ and $C$ and by decreasing the step size from the current $45^{\circ}$.

The various field experiments clearly demonstrate that the raft is capable of navigating in a robust fashion and detecting the presence of fish reliably. The primary results in localizing the fish precisely are promising, and our future work is directed towards improving this capability further.

\section{CONCLUSION}

In this paper, we presented progress in building a robotic sensor network for monitoring carp in Minnesota lakes. Field experiments demonstrate that a light-weight, inexpensive robotic system has the potential for tremendous utility in environmental monitoring by allowing scientists to collect data over long periods of time from hard-to-reach locations.

We are working on improving our system in a number of directions. Here, we present a brief overview of our agenda.

Energy: To improve the current 5 hours battery life, we are planning to add solar panels to the system. Environmental scientists frequently require collecting data in the night and daybreak. Hence, the system cannot completely rely on solar panels as power source. This opens up interesting algorithmic questions regarding energy harvesting during search and tracking and energy efficient operation.

Localization accuracy: As described in the paper, signal strength and bearing information individually are insufficient for accurate localization of the fish. We are investigating ways for augmenting both the information in a unified manner for better localization.

Autonomous navigation: Currently, the system follows GPS waypoints predefined by the user, while searching for fish in the lake. In the next phase, we will focus on adaptive, autonomous behavior. We are currently working on search strategies that maximize the probability of locating the fish. We are also working on designing strategies for tracking individual fish after locating it.

Multi-raft systems: For localizing a fish with a single raft, two or more readings from different locations are needed. By the time the raft moves from one point to the other, the fish can move, and the resulting localization may not be precise. However, if there are two or more rafts working in coordination, the fish could be localized at a single instance of time with one reading from each raft. This would give better localization. Multiple rafts could also prove useful in searching the lake for presence of fish and while tracking a single fish. The design of inexpensive, easy-to-build systems (such as the one presented here) is especially important for building real-life multi-robot systems.

Acknowledgments: The authors are grateful to Prof. Peter Sorensen and the members of his lab for numerous useful discussions and sharing equipment. This work is supported in part by NSF Projects 0917676, 0907658 and 0936710, and a fellowship from the Institute on the Environment at the University of Minnesota.

\section{REFERENCES}

[1] K. Akkaya and M. Younis, "A survey on routing protocols for wireless sensor networks," Ad Hoc Networks, vol. 3, no. 3, pp. 325-349, 2005.

[2] "Vineyard uses sensor network to fine-tune irrigation," NetworkWorld, 2009. [Online]. Available: http://www.networkworld.com/newsletters/ wireless/2009/030209wireless2.html

[3] H. Ferreira, A. Martins, A. Dias, C. Almeida, J. Almeida, and E. Silva, "Roaz autonomous surface vehicle design and implementation," ROBOTICA, 2006.

[4] A. Subramanian, X. Gong, J. Riggins, D. Stilwell, and C. Wyatt, "Shoreline mapping using an omni-directional camera for autonomous surface vehicle applications," in OCEANS 2006, Sept. 2006, pp. 1-6.

[5] E. Steimle and M. Hall, "Unmanned Surface Vehicles as Environmental Monitoring and Assessment Tools," OCEANS 2006, pp. 1-5, 2006.

[6] D. Blidberg, "The development of autonomous underwater vehicles (AUVs); a brief summary," in IEEE ICRA, 2001.

[7] M. Dunbabin, J. Roberts, K. Usher, G. Winstanley, and P. Corke, "A hybrid AUV design for shallow water reef navigation," in Robotics and Automation, 2005. ICRA 2005. Proceedings of the 2005 IEEE International Conference on, 2005, pp. 2105-2110.

[8] M. Kumagai, T. Ura, Y. Kuroda, and R. Walker, "A new autonomous underwater vehicle designed for lake environment monitoring," $A d$ vanced Robotics, vol. 16, no. 1, pp. 17-26, 2002.

[9] C. Eriksen, T. Osse, R. Light, T. Wen, T. Lehman, P. Sabin, J. Ballard, and A. Chiodi, "Seaglider: a long-range autonomous underwater vehicle foroceanographic research," IEEE Journal of Oceanic Engineering, vol. 26, no. 4, pp. 424-436, 2001.

[10] E. Levine and R. Lueck, "Turbulence measurement from an autonomous underwater vehicle," Journal of Atmospheric and Oceanic Technology, vol. 16, no. 11, pp. 1533-1544, 1999.

[11] G. Marani, S. Choi, and J. Yuh, "Underwater autonomous manipulation for intervention missions AUVs," Ocean Engineering, vol. 36, no. 1, pp. 15-23, 2009.

[12] G. Sukhatme, A. Dhariwal, B. Zhang, C. Oberg, B. Stauffer, and D. Caron, "Design and development of a wireless robotic networked aquatic microbial observing system," Environmental Engineering Science, vol. 24, no. 2, pp. 205-215, 2007. 